\title{
EXCLUDING SUBDIVISIONS OF INFINITE CLIQUES
}

\author{
NEIL ROBERTSON, P. D. SEYMOUR, AND ROBIN THOMAS
}

Abstract. For every infinite cardinal $\kappa$ we characterize graphs not containing a subdivision of $K_{\kappa}$.

\section{INTRODUCTION}

In this paper graphs may be infinite, and may have loops and multiple edges. A graph $G$ is a subdivision of a graph $H$ if $G$ can be obtained from $H$ by replacing the edges of $H$ by internally disjoint paths joining the same ends. Let $G$ be a graph. A tree-decomposition of $G$ is a pair $(T, W)$, where $T$ is a tree (a connected graph with no circuits) and $W=\left(W_{t}: t \in V(T)\right)$ is such that

(W1) $\bigcup_{t \in V(T)} W_{t}=V(G)$, and every edge of $G$ has both ends in some $W_{t}$, and

(W2) if $t^{\prime}$ lies on the path of $T$ between $t$ and $t^{\prime \prime}$, then $W_{t} \cap W_{t^{\prime \prime}} \subseteq W_{t^{\prime}}$. If $\kappa$ is a cardinal, we say that $(T, W)$ has width $<\kappa$ if $\left|W_{t}\right|<\kappa$ for every $t \in V(T)$, and

$$
\left|\bigcup_{i=1}^{\infty} \bigcap_{j \geq i} W_{t_{j}}\right|<\kappa
$$

for every infinite path $t_{1}, t_{2}, \ldots$ in $T$. We shall prove the following result for excluding a subdivision of $K_{\aleph_{0}}$, the countable clique.

(1.1) A graph $G$ contains no subgraph isomorphic to a subdivision of $K_{\aleph_{0}}$ if and only if $G$ admits a tree-decomposition of width $<\aleph_{0}$.

One cannot hope for an analogous theorem for uncountable cardinals because of the following [4].

(1.2) For every cardinal $\kappa$ there exists a graph $G$ with no subgraph isomorphic to a subdivision of $K_{\aleph_{1}}$ such that for every tree-decomposition $(T, W)$ of $G$ there exists $t \in V(T)$ with $\left|W_{t}\right| \geq \kappa$.

However, the next best weakening works, namely "well-founded tree-decomposition," which we now introduce. A well-founded tree is a nonempty partially ordered set $T=(V, \leq)$ such that for every two elements $t_{1}, t_{2} \in V$ their infimum exists and such that the set $\left\{t^{\prime} \in V: t^{\prime}<t\right\}$ is well-ordered for every

Received by the editors March 30, 1990.

1980 Mathematics Subject Classification (1985 Revision). Primary 05 C75.

The first and third authors' research was performed under a consulting agreement with Bellcore.

The first author was also supported by NSF under Grant No. DMS-8903132.

The third author was also supported by DIMACS Center, Rutgers University, New Brunswick, New Jersey. 
$t \in V$. It follows that $T$ has a minimum element, called the root and denoted by $\operatorname{root}(T)$. We write $V(T)=V$ and call the elements of $V(T)$ the vertices of $T$. If $\lambda$ is an ordinal we say that $T$ is $<\lambda$ high if every chain in $T$ has order type $<\lambda$. For $t_{1}, t_{2} \in V(T)$ we define $T\left[t_{1}, t_{2}\right]$ to be the set of all $t \in V(T)$ such that $\inf \left(t_{1}, t_{2}\right) \leq t$, and either $t \leq t_{1}$ or $t \leq t_{2}$. For $t, t^{\prime} \in V(T)$ we say that $t^{\prime}$ is a successor of $t$, and that $t$ is a predecessor of $t^{\prime}$, if $t<t^{\prime}$ and there is no $t^{\prime \prime} \in V(T)-\left\{t, t^{\prime}\right\}$ with $t \leq t^{\prime \prime} \leq t^{\prime}$.

A well-founded tree-decomposition of a graph $G$ is a pair $(T, W)$, where $T$ is a well-founded tree and $W=\left(W_{t}: t \in V(T)\right)$ satisfies

(W1) $\bigcup_{t \in V(T)} W_{t}=V(T)$, and every edge of $G$ has both ends in some $W_{t}$,

(W2) if $t^{\prime} \in T\left[t, t^{\prime \prime}\right]$ then $W_{t} \cap W_{t^{\prime \prime}} \subseteq W_{t^{\prime}}$, and

(W3) if $C \subseteq V(T)$ is a chain and $c=\sup C \in V(T)$, then $\bigcap_{t \in C} W_{t} \subseteq W_{c}$.

We say that $(T, W)$ has width $<\kappa$ if $\left|\bigcup_{t \in C} \bigcap\left\{W_{t^{\prime}}: t^{\prime} \in C, t^{\prime} \geq t\right\}\right|<\kappa$ for every chain $C \subseteq V(T)$. It follows that if $(T, W)$ has width $<\kappa$ then $\left|W_{t}\right|<\kappa$ for every $t \in V(T)$. We say that $(T, W)$ is $<\kappa$ high if $T$ is $<\kappa$ high.

We say that a well-founded tree $T$ is graph-theoretic if every chain in $T$ has order type $\leq \omega$. Let $R$ be a tree and let $r \in V(R)$. We define $t_{1} \leq t_{2}$ for $t_{1}, t_{2} \in V(R)$ to mean that $t_{1}$ lies on the path between $r$ and $t_{2}$. It is easily seen that $T=(V(R), \leq)$ is a graph-theoretic well-founded tree and that every graph-theoretic well-founded tree arises this way. Moreover, $T\left[t_{1}, t_{2}\right]$ is the set of all vertices of $R$ which lie on the path between $t_{1}$ and $t_{2}$ in $R$. We say that a well-founded tree-decomposition $(T, W)$ is graph-theoretic if $T$ is graph-theoretic. Thus we have proved

(1.3) Let $\kappa$ be a cardinal. A graph $G$ admits a graph-theoretic well-founded tree-decomposition of width $<\kappa$ if and only if $G$ admits a tree-decomposition of width $<\kappa$.

The true version of (1.1) for larger cardinals is the following.

(1.4) Let $\kappa$ be an infinite cardinal and let $G$ be a graph. Then the following two conditions are equivalent:

(i) $G$ contains no subgraph isomorphic to a subdivision of $K_{\kappa}$,

(ii) $G$ admits a well-founded tree-decomposition of width $<\kappa$.

If $\kappa=\aleph_{0}$ then the tree-decomposition in (ii) can be chosen graph-theoretic. If $\kappa$ is regular and uncountable, then (i) and (ii) are equivalent to

(iii) $G$ admits a well-founded tree-decomposition of width $<\kappa$ which is $<\kappa$ high.

By (1.3), (1.4) implies (1.1). Notice that if $\kappa$ is regular and $(T, W)$ is a treedecomposition of a graph $G$ which is $<\kappa$ high, then $(T, W)$ has width $<\kappa$ if and only if $\left|W_{t}\right|<\kappa$ for every $t \in V(T)$. The equivalence of (i) and (iii) for regular uncountable cardinals is similar to (and interderivable with) a result independently obtained by Diestel [2]. Diestel's theorem generalizes a theorem of Halin [3].

There are other conditions that are equivalent to the conditions of (1.4) when $\kappa$ is regular uncountable. We now introduce two of them; others can be found in [7].

A linear decomposition of a graph $G$ is a pair $(L, X)$, where $L$ is a (Dedekind) complete linearly ordered set and $X=\left(X_{l}: l \in L\right)$ is such that

(L1) $\bigcup_{l \in L} X_{l}=V(G)$, and every edge of $G$ has both ends in some $X_{l}$, 
(L2) if $l \leq l^{\prime} \leq l^{\prime \prime}$, then $X_{l} \cap X_{l^{\prime \prime}} \subseteq X_{l^{\prime}}$, and

(L3) $\bigcap_{i \in I} X_{i} \subseteq X_{\inf (I)} \cap X_{\sup (I)}$ for every nonempty interval $I \subseteq L$.

We say that $(L, X)$ has width $<\kappa$ if $\left|X_{l}\right|<\kappa$ for every $l \in L$. Let us remark that the requirement that $L$ be complete is not restrictive, because any "incomplete" decomposition can be completed in the obvious way. Linear decompositions are motivated by path-decompositions from [5], and their relation to excluding infinite trees is studied in [8].

Finally, we introduce the following generalization of stoppages from [1]. Let $G$ be a graph. A cut in $G$ is an ordered pair $(A, B)$ of subsets of $V(G)$ such that $A \cup B=V(G)$ and there is no edge between $A-B$ and $B-A$. The order of $(A, B)$ is $|A \cap B|$. Now let $\kappa$ be a cardinal. A stoppage of order $\kappa$ in a graph $G$ is a set $\mathscr{S}$ of cuts, all of order $<\kappa$, such that

(i) if $(A, B)$ is a cut in $G$ of order $<\kappa$, then $\mathscr{S}$ contains one of $(A, B)$, $(B, A)$,

(ii) if $\left(A_{1}, B_{1}\right),\left(A_{2}, B_{2}\right) \in \mathscr{S}$, then $\left(G \mid A_{1}\right) \cup\left(G \mid A_{2}\right) \neq G$ (where $G \mid A$ is the restriction of $G$ to $A$ ), and

(iii) if $\mathscr{M} \subseteq \mathscr{S}$ is a chain of cuts (that is, for $\left(A_{1}, B_{1}\right),\left(A_{2}, B_{2}\right) \in \mathscr{M}$ either $A_{1} \subseteq A_{2}$ and $B_{1} \supseteq B_{2}$, or $A_{1} \supseteq A_{2}$ and $\left.B_{1} \subseteq B_{2}\right)$ and for

$$
A=\bigcup\left\{A^{\prime}:\left(A^{\prime}, B^{\prime}\right) \in \mathscr{M}\right\}, \quad B=\bigcap\left\{B^{\prime}:\left(A^{\prime}, B^{\prime}\right) \in \mathscr{M}\right\}
$$

the order of $(A, B)$ is $<\kappa$, then $(A, B) \in \mathscr{S}$.

We will refer to (i), (ii), (iii) as the stoppage axioms.

The following result extends (1.4) for $\kappa$ regular uncountable.

(1.5) Let $G$ be a graph and let $\kappa$ be a regular uncountable cardinal. Then the following conditions are equivalent:

(i) $G$ has no subgraph isomorphic to a subdivision of $K_{\kappa}$,

(ii) $G$ admits $a<\kappa$ high well-founded tree-decomposition of width $<\kappa$,

(iii) $G$ admits a linear decomposition of width $<\kappa$,

(iv) $G$ has no stoppage of order $\geq \kappa$.

Theorem (1.5) is false for $\kappa=\aleph_{0}$, because it is shown in [8] that if $\kappa=\aleph_{0}$ then (iii) is equivalent to not containing a subgraph isomorphic to a subdivision of the $\aleph_{0}$-branching tree. The assumption that $\kappa$ is regular cannot be dropped either, because of the following (we do not know whether there is a similar counterexample when $\operatorname{cf}(\kappa)>\omega)$.

(1.6) There exists a graph which contains no subgraph isomorphic to a subdivision of $K_{\aleph_{\omega}}$ and which has no linear decomposition of width $<\aleph_{\omega}$.

The paper is organized as follows: In $\S 2$ we prove (1.4) and in $\S 3$ we prove (1.5) and (1.6). We end this section with the following lemma, a relative of (3.4) from [6].

(1.7) Let $G$ be a graph, let $F$ be the vertex-set of a connected subgraph of $G$, let $(T, W)$ be a well-founded tree-decomposition of $G$ and let $t_{1}, t_{2} \in V(T)$ be such that $W_{t_{1}} \cap F \neq \varnothing \neq W_{t_{2}} \cap F$. If $t, t^{\prime} \in T\left[t_{1}, t_{2}\right]$ are such that $t \leq t^{\prime}$ and there is no $t^{\prime \prime} \in V(T)$ with $t<t^{\prime \prime}<t^{\prime}$ then $W_{t} \cap W_{t^{\prime}} \cap F \neq \varnothing$. In particular, if $t \in T\left[t_{1}, t_{2}\right]$ then $W_{t} \cap F \neq \varnothing$. 
Proof. We proceed by induction on $|V(P)|$, where $P$ is the shortest path connecting $W_{t_{1}}$ and $W_{t_{2}}$ with $V(P) \subseteq F$. If $|V(P)|=1$ the result follows from (W2). If $|V(P)| \geq 2$ let $u \in V(P) \cap W_{t_{1}}$ be an endvertex of $P$, and let $v \in V(P)$ be the neighbor of $u$ in $P$. By (W1) there is $r \in V(T)$ such that $u, v \in W_{r}$. It is easy to see tha: either $t, t^{\prime} \in T\left[t_{1}, r\right]$ or $t, t^{\prime} \in T\left[r, t_{2}\right]$. In the former case $u \in W_{t} \cap W_{t^{\prime}} \cap F$ by (W2), and in the latter one the result follows from the induction hypothesis applied to $r, t_{2}$ and $F$.

\section{GREEDY METHOD}

In this section we prove (1.4). First we shall prove that (ii) implies (i).

(2.1) Let $\kappa$ be a cardinal, let $G$ be a graph and let $(T, W)$ be a well-founded tree-decomposition of $G$ of width $<\kappa$. Then $G$ contains contains no subgraph isomorphic to a subdivision of $K_{\kappa}$.

Proof. Let $(T, W)$ be as stated in (2.1). Suppose for a contradiction that $G$ contains a subgraph $H$ isomorphic to a subdivision of $K_{\kappa}$, and let $V \subseteq V(H)$ be the set of vertices corresponding to vertices of $K_{\kappa}$. For each $v \in V$, let $t(v)$ be the minimal $t \in V(T)$ with $v \in W_{t}$ (it is unique by (W2)).

(1) If $v_{1}, v_{2} \in V$ then either $t\left(v_{1}\right) \leq t\left(v_{2}\right)$ or $t\left(v_{2}\right) \leq t\left(v_{1}\right)$.

For let $t=\inf \left(t\left(v_{1}\right), t\left(v_{2}\right)\right)$. There are $\kappa$ paths of $H$ and hence of $G$, between $v_{1}$ and $v_{2}$, mutually disjoint except for $v_{1}$ and $v_{2}$, and all passing through $W_{t}$ by (1.7). Since $\left|W_{t}\right|<\kappa$ it follows that one of $v_{1}, v_{2} \in W_{t}$, and so either $t=t\left(v_{1}\right)$ or $t=t\left(v_{2}\right)$. The claim follows.

(2) If $v_{1}, v_{2} \in V$ and $t\left(v_{1}\right) \leq t\left(v_{2}\right)$ then $v_{1} \in W_{t}$ for all $t$ with $t\left(v_{1}\right) \leq t \leq$ $t\left(v_{2}\right)$.

For suppose not. Choose $t_{2}$ with $t\left(v_{1}\right) \leq t_{2} \leq t\left(v_{2}\right)$ minimal such that $v_{1} \notin W_{t_{2}}$. Since $v_{1} \in W_{t}$ for all $t$ with $t\left(v_{1}\right) \leq t<t_{2}$, it follows that $t_{2} \neq \sup \left\{t: t\left(v_{1}\right) \leq t<t_{2}\right\}$ by (W3), and so there exists a predecessor $t_{1}$ of $t_{2}$. By (1.7), each of the $\kappa$ internally disjoint paths of $H$ between $v_{1}$ and $v_{2}$ passes through $W_{t_{1}} \cap W_{t_{2}}$, and so one of $v_{1}, v_{2}$ belongs to $W_{t_{1}} \cap W_{t_{2}}$. But $v_{1} \notin W_{t_{2}}$, and $v_{2} \notin W_{t_{1}}$ since $t_{1}<t_{2} \leq t\left(v_{2}\right)$. This is a contradiction, and (2) follows.

Let $C=\{t \in V(T): t \leq t(v)$ for some $v \in V\}$.

(3) $C$ is a chain, and $V \subseteq \bigcup_{t \in C} \bigcap\left\{W_{t^{\prime}}: t^{\prime} \in C, t^{\prime} \geq t\right\}$.

For $C$ is a chain by (1). Let $v \in V$, and let $t^{\prime} \in C, t^{\prime} \geq t(v)$. Choose $v^{\prime} \in V$ with $t^{\prime} \leq t\left(v^{\prime}\right)$. Then $t(v) \leq t\left(v^{\prime}\right)$ and by (2), $v \in W_{t^{\prime}}$. Hence $v \in \bigcap\left\{W_{t^{\prime}}: t^{\prime} \in C, t^{\prime} \geq t(v)\right\}$ and the claim follows.

But $|V|=\kappa$, and so (3) contradicts the fact that $(T, W)$ has width $<\kappa$.

Next we prove the rest of (1.4). We first prove that every graph $G$ admits a "standard decomposition" and then prove that if $G$ is as in (1.4)(i) then this decomposition satisfies the conclusion of (1.4)(ii). If $X$ is a set we put $\|X\|=0$ if $X$ is finite, and $\|X\|=|X|$ otherwise. If $G$ is a graph and $K \subseteq V(G)$, then $N(K)$ is the set of all vertices in $V(G)-K$ which are adjacent to a vertex in $K$, $G \backslash K$ is the graph obtained from $G$ by deleting the vertices of $K$ and all edges incident with these vertices, and a $K$-flap is the set of vertices of a component of $G \backslash K$.

Let $(T, W)$ be a well-founded tree-decomposition of a graph $G$. We say 
that $(T, W)$ is a standard decomposition of $G$ if

(S1) $W_{\operatorname{root}(T)}=\varnothing$,

(S2) if $t^{\prime}$ is a successor of $t$ in $T$, then $\left\|W_{t^{\prime}}\right\| \leq\left\|W_{t}\right\|$ and $W_{t^{\prime}}-W_{t} \neq \varnothing$,

(S3) for every chain $C$ in $T$ which does not have a maximal element, any two nonadjacent vertices in $W_{C}:=\bigcup_{t \in C} \bigcap\left\{W_{t^{\prime}}: t^{\prime} \in C, t^{\prime} \geq t\right\}$ are joined in $G$ by $\left\|W_{C}\right\|$ internally disjoint paths, and if $C$ has a supremum, say $t$, then $W_{t}=W_{C}$ and $t$ has at least one successor,

(S4) for every $t \in V(T)$, every $W_{t}$-flap of $G$ which intersects $\bigcup_{t \leq t^{\prime}} W_{t^{\prime}}$ is intersected by $W_{t^{\prime}}$ for some successor $t^{\prime}$ of $t$.

(2.2) Every graph admits a standard decomposition.

Proof. Let $G$ be a graph. Let $u, v \in X \subseteq V(G)$ and let $\mu$ be a cardinal. If there exists a set $Y \subseteq X-\{u, v\}$ with $\|Y\| \leq \mu$ which meets every path $P$ joining $u, v$ with $V(P) \subseteq X$, then we let $\Theta(u, v, X, \mu)$ be one such set $Y$, and if not we define $\Theta(u, v, X, \mu)=\varnothing$.

For some ordinal $\lambda$, we shall construct a transfinite sequence $W_{\alpha} \quad(\alpha \leq \lambda)$ of subsets of $V(G)$ satisfying

(1) $\varnothing=W_{0} \subseteq W_{1} \subseteq \cdots \subseteq W_{\alpha} \subseteq \cdots \subseteq W_{\lambda}=V(G)$.

Also, for each $\alpha<\lambda$ we shall construct a partition $\mathscr{K}_{\alpha}$ of $V(G)-W_{\alpha}$ into nonempty sets satisfying

(2) for all $\alpha<\lambda$ if $K, K^{\prime} \in \mathscr{K}_{\alpha}$ are distinct then no vertex of $K$ has a neighbor in $K^{\prime}$, and

(3) for $\alpha \leq \beta<\lambda$, every member of $\mathscr{K}_{\beta}$ is a subset of a member of $\mathscr{K}_{\alpha}$.

Third, for each $\alpha<\lambda$ and each $K \in \mathscr{K}_{\alpha}$ we shall construct a subset $W_{(K, \alpha)}$ of $K \cup W_{\alpha}$.

The inductive definition is as follows. Let $W_{0}=\varnothing, \mathscr{K}_{0}=\{V(G)\}$, and $W_{(V(G), 0)}=\varnothing$. Suppose that for some ordinal $\alpha$ we have defined $W_{\beta}, \mathscr{K}_{\beta}$ and $W_{(K, \beta)}\left(K \in \mathscr{K}_{\beta}\right)$ for all $\beta<\alpha$. Now we wish to define $W_{\alpha}, \mathscr{K}_{\alpha}, W_{(K, \alpha)}$ $\left(K \in \mathscr{K}_{\alpha}\right)$. Let

(4) $W_{\alpha}=\bigcup\left\{W_{(K, \beta)}: \beta<\alpha, K \in \mathscr{K}_{\beta}\right\}$.

If $W_{\alpha}=V(G)$ we set $\lambda=\alpha$ and stop. Otherwise, there are two cases.

If $\alpha$ is a successor ordinal, say $\alpha=\beta+1$, let $\mathscr{K}_{\alpha}$ be the set of all $W_{\alpha}$-flaps of $G$. For each $K \in \mathscr{K}_{\alpha}$ let $L \in \mathscr{K}_{\beta}$ include $K$, let $w \in K$ be arbitrary, let $Z=\{w\} \cup N(K)$, and let

$$
W_{(K, \alpha)}=Z \cup \bigcup\left\{\Theta\left(u, v, K \cup N(K),\left\|W_{(L, \beta)}\right\|\right): u, v \in Z\right\} .
$$

Now, let $\alpha$ be a limit ordinal. For each $u \in V(G)-W_{\alpha}$ and each $\beta<\alpha$, let $K_{\beta}(u)$ be the member of $\mathscr{K}_{\beta}$ containing $u$; then, by (3),

$$
K_{0}(u) \supseteq K_{1}(u) \supseteq \cdots \supseteq K_{\beta}(u) \supseteq \cdots .
$$

Let $K(u)=\bigcap_{\beta<\alpha} K_{\beta}(u)$, and let $\mathscr{K}_{\alpha}=\left\{K(u): u \in V(G)-W_{\alpha}\right\}$. For $u \in$ $V(G)-W_{\alpha}$, let

$$
W_{(K(u), \alpha)}=\bigcup_{\beta<\alpha} \bigcap_{\beta<\gamma<\alpha} W_{\left(K_{\gamma}(u), \gamma\right)} .
$$

This completes the inductive definition. It is easy to see that the ordinal $\lambda$ indeed exists, that $W_{(K, \alpha)} \subseteq K \cup W_{\alpha}$ for every $\alpha<\lambda$ and every $K \in \mathscr{K}_{\alpha}$, and that (1), (2), (3) are satisfied. 
We put $V(T)=\left\{(K, \alpha): K \in \mathscr{K}_{\alpha}, \alpha \leq \lambda\right\}$ and define $(K, \alpha) \leq\left(K^{\prime}, \alpha^{\prime}\right)$ if $K \supseteq K^{\prime}$ and $\alpha \leq \alpha^{\prime}$. We put $T=(V(T), \leq)$. It follows easily that $T$ is a wellfounded tree. Let $W=\left(W_{(K, \alpha)}:(K, \alpha) \in V(T)\right)$. We shall show that $(T, W)$ is the desired standard decomposition, but we need several observations before we do so.

(5) $N(K) \subseteq W_{(K, \alpha)}$ for every $K \in \mathscr{K}_{\alpha}$ and every ordinal $\alpha<\lambda$.

We prove this by transfinite induction on $\alpha$. The statement is obviously satisfied when $\alpha=0$, so let $\alpha$ be an ordinal and assume that (5) holds for all $\beta<\alpha$. Let $K \in \mathscr{K}_{\alpha}$ and let $v \in N(K)$. If $\alpha$ is a successor ordinal then $v \in W_{(K, \alpha)}$ by the definition of $W_{(K, \alpha)}$ so let $\alpha$ be a limit ordinal. Let $u$ be a neighbor of $v$ in $K$. Define $K_{\beta}(u)(\beta<\alpha)$ as before; then $K=\bigcap_{\beta<\alpha} K_{\beta}(u)$. Since $v \notin K$, there exists $\beta<\alpha$ with $v \notin K_{\beta}(u)$, and hence with $v \notin K_{\gamma}(u)$ for $\beta<\gamma<\alpha$. Since $u, v$ are adjacent and $u \in K_{\gamma}(u)$, it follows that $v \in N\left(K_{\gamma}(u)\right) \subseteq W_{\left(K_{\gamma}(u), \gamma\right)}$ for $\beta<\gamma<\alpha$, by the inductive hypothesis; and thus $v \in W_{(K, \alpha)}$ by the definition of $W_{(K, \alpha)}$. This proves (5).

(6) For $\alpha \leq \beta<\lambda$, if $K \in \mathscr{K}_{\alpha}$ and $L \in \mathscr{K}_{\beta}$ and $L \subseteq K$ then $W_{(L, \beta)} \subseteq$ $W_{(K, \alpha)} \cup K$.

We prove this by transfinite induction on $\beta$. We may assume that $\alpha<\beta$. If $\beta$ is a successor, $\beta=\gamma+1$ say, let $(L, \beta)$ be a successor of $(M, \gamma)$. Then

$$
W_{(L, \beta)} \subseteq L \cup N(L) \subseteq M \cup N(M) \subseteq M \cup W_{(M, \gamma)}
$$

by (5), and $W_{(M, \gamma)} \subseteq W_{(K, \alpha)} \cup K$ by the induction hypothesis. It follows that $W_{(L, \beta)} \subseteq M \cup W_{(K, \alpha)} \cup K \subseteq W_{(K, \alpha)} \cup K$, as required. Now assume that $\beta$ is a limit ordinal. Let $v \in W_{(L, \beta)}$. By definition of $W_{(L, \beta)}$ there exists $(M, \gamma)$ with $(K, \alpha) \leq(M, \gamma)<(L, \beta)$ with $v \in W_{(M, \gamma)}$. By the induction hypothesis, $W_{(M, \gamma)} \subseteq W_{(K, \alpha)} \cup K$, and so $v \in W_{(K, \alpha)} \cup K$. The result follows.

Let $v \in V(G)$. A pair $(K, \alpha) \in V(T)$ is called a nest of $v$ if $v \in W_{(K, \alpha)}$ and for every ordinal $\beta<\alpha$ there is no $L \in \mathscr{K}_{\beta}$ such that $v \in W_{(L, \beta)}$. Since $W_{\lambda}=V(G)$, it follows from (4) with $\alpha=\lambda$ that there is a nest of $v$, for every $v \in V(G)$.

(7) Let $(K, \alpha)$ be a nest of $v \in V(G)$. Then $v \in K$.

For it follows from the minimality of $\alpha$ and (4) that $v \notin W_{\alpha}$. Since $v \in W_{(K, \alpha)} \subseteq K \cup W_{\alpha}$, the claim follows.

(8) If $(K, \alpha)$ is a nest of $v \in W_{(L, \beta)}$, then $(K, \alpha) \leq(L, \beta)$.

For $\alpha \leq \beta$, by the minimality of $\alpha$. Let $K^{\prime} \in \mathscr{K}_{\alpha}$ with $L \subseteq K^{\prime}$. Since $v \in K$ by (7), and $v \in W_{(L, \beta)} \subseteq W_{\left(K^{\prime}, \alpha\right)} \cup K^{\prime} \subseteq W_{\alpha} \cup K^{\prime}$ by (6), we deduce that $K \cap\left(W_{\alpha} \cap K^{\prime}\right) \neq \varnothing$, and so $K=K^{\prime}$. The result follows.

From (8) we deduce that the nest of $v$ is unique.

(9) $(T, W)$ is a well-founded tree-decomposition.

We must verify (W1)-(W3). We start with (W1). Certainly $\bigcup_{t \in V(T)} W_{t}=$ $V(T)$, by (4) with $\alpha=\lambda$. Let $e$ be an edge of $G$ with endvertices $u, v$, say. Let $(K, \alpha)$ be the nest of $u$ and let $(L, \beta)$ be the nest of $v$. We may assume that $\alpha \leq \beta$. It follows from (7) that $u \in K$ and $v \in L$, and since they are adjacent we deduce that $L \subseteq K$. If $\alpha=\beta$ then $K=L$ and we are done, and so we assume that $\alpha<\beta$. Then $u \notin L$ since $W_{(K, \alpha)} \subseteq W_{\alpha+1} \subseteq W_{\beta}$ and $L \cap W_{\beta}=\varnothing$. Hence $u \in N(L)$ and so $u \in W_{(L, \beta)}$ by (5), as desired. This proves (W1).

To verify (W2) let $v \in W_{(K, \alpha)} \cap W_{(L, \beta)}$. First let $(K, \alpha)<(M, \gamma)<(L, \beta)$. Then $L \subseteq M \subseteq K$ and $v \notin M$ because $M$ is disjoint from $W_{(K, \alpha)}$. Therefore, 
$v \in W_{(L, \beta)}-M \subseteq W_{(M, \gamma)}$ by (6). Hence (W2) holds for $t, t^{\prime}, t^{\prime \prime}$ if $t<t^{\prime}<t^{\prime \prime}$. Now let $K \cap L=\varnothing$. Let $(N, \delta)$ be the nest of $v$; then $(N, \delta) \leq(K, \alpha)$ and $(N, \delta) \leq(L, \beta)$ by $(8)$. This together with the above shows that $v \in W_{(M, \gamma)}$ for every $(M, \gamma) \in T[(K, \alpha),(L, \beta)]$.

Finally, condition (W3) follows directly from the construction. This completes the proof of (9).

(10) If $(K, \alpha),(L, \alpha+1) \in V(T)$ and $(L, \alpha+1)$ is a successor of $(K, \alpha)$, then $N(L) \subseteq W_{(K, \alpha)}$.

For let $v \in N(L)$ and let $(M, \beta)$ be the nest of $v$. Since $v \in N(L) \subseteq$ $W_{\alpha+1}$ by (2), it follows from (4) that $\beta \leq \alpha$. If $\beta=\alpha$ then $K=M$ and $v \in W_{(M, \beta)}=W_{(K, \alpha)}$, while if $\beta<\alpha$ then $v \notin K$ because $K \cap W_{(M, \beta)}=\varnothing$, and hence $v \in N(K) \subseteq W_{(K, \alpha)}$ by (5).

(11) $(T, W)$ is a standard decomposition.

We must verify (S1)-(S4). Condition (S1) is clear. To see (S2) let $(K, \alpha+1)$ be a successor of $(L, \alpha)$. Then $N(K) \subseteq W_{(L, \alpha)}$ by (10), and by the construction

$$
W_{(K, \alpha+1)}=Z \cup \bigcup\left\{\Theta\left(u, v, K \cup N(K),\left\|W_{(L, \alpha)}\right\|\right): u, v \in Z\right\},
$$

where $Z=\{w\} \cup N(K)$ and $w \in K$. Thus $w \in W_{(K, \alpha+1)}-W_{(L, \alpha)} \neq \varnothing$, and

$$
\left\|W_{(K, \alpha+1)}\right\| \leq\|Z\|+\|Z\|^{2} \cdot\left\|W_{(L, \alpha)}\right\| \leq\left\|W_{(L, \alpha)}\right\|,
$$

because $\|Z\| \leq\left\|W_{(L, \alpha)}\right\|$. To prove (S3) let $C$ be a chain in $T$ which does not have a maximal element, and let $W_{C}=\bigcup_{t \in C} \bigcap\left\{W_{t^{\prime}}: T^{\prime} \in C, t^{\prime} \geq t\right\}$. Suppose for a contradiction that $u, v \in W_{C}$ are separated by a cutset, say $A$, of cardinality $<\left\|W_{C}\right\|$. It follows that $\left\|W_{C}\right\|=\left|W_{C}\right| \geq \aleph_{0}$. Then there exists $(K, \alpha) \in C$ such that $\left|W_{(K, \alpha)}\right| \geq|A|$ and $u, v \in W_{t}$ for every $t \in C$ with $t \geq(K, \alpha)$. Let $(L, \alpha+1) \in C$ be the successor of $(K, \alpha)$, and let $(M, \alpha+2) \in$ $C$ be the successor of $(L, \alpha+1)$. It follows that $u, v \in N(L) \cap N(M)$. By construction $W_{(L, \alpha+1)}-\{u, v\}$ meets every path $P$ joining $u$ and $v$ with $V(P) \subseteq L \cup N(L)$, but that contradicts the fact that $u, v \in N(M)$ since $M \cap W_{(L, \alpha+1)}=\varnothing$ and $M$ is a $W_{\alpha+2}$-flap. Hence $u, v$ are not separated by a cutset of size $<\left\|W_{C}\right\|$, and the first part of (S3) follows. Now if $C$ has a supremum, say $t=(K, \beta)$, then $W_{t}=W_{C}$ follows directly from the construction. It also follows that $\beta$ is a limit ordinal, and $\beta<\lambda$. Since $W_{(M, \beta)} \subseteq W_{\beta}$ for all $M \in \mathscr{K}_{\beta}$ from the construction, and hence $W_{\beta+1}=W_{\beta}$, it follows that $K$ includes a $W_{\beta+1}$-flap $L$ since $K \neq \varnothing$, and hence $(L, \beta+1)$ is a successor of $(K, \beta)$. This proves (S3).

To prove (S4) let $t=(K, \alpha)$ and let $F$ be a $W_{t}$-flap which intersects $\bigcup_{t \leq t^{\prime}} W_{t^{\prime}}$. We claim that $F \cap W_{\alpha+1}=\varnothing$. For if, say, $F \cap W_{(L, \beta)} \neq \varnothing$, where $\beta \leq \alpha$ then $t \nless(L, \beta)$ and so $F \cap W_{t} \neq \varnothing$ by (1.7), a contradiction. Hence $F \cap W_{\alpha+1}=\varnothing$. It follows that $F$ is a $W_{\alpha+1}$-flap and thus $F \in \mathscr{K}_{\alpha+1}$. But $F \cap W_{(F, \alpha+1)} \neq \varnothing$ by the construction, as desired.

The following is an easy lemma and the proof is left to the reader.

(2.3) Let $G$ be a graph and let $\kappa$ be an infinite cardinal. If there exists a set $X \subseteq V(G)$ with $|X| \geq \kappa$ such that every two nonadjacent vertices of $X$ are joined $\bar{b} y \boldsymbol{\kappa}$ internally disjoint paths, then $G$ contains a subgraph $H$ isomorphic to a subdivision of $K_{\kappa}$ in such a way that every vertex of $H$ which corresponds to a vertex of $K_{\kappa}$ belongs to $X$. 
The following result implies (i) $\Rightarrow$ (ii) of (1.4), because (1.4) obviously holds for finite graphs.

(2.4) Let $\kappa$ be an infinite cardinal, let $G$ be an infinite graph which contains no subgraph isomorphic to a subdivision of $K_{\kappa}$ and let $(T, W)$ be a standard decomposition of $G$. Then $|V(T)| \leq|V(G)|$ and $(T, W)$ has width $<\kappa$.

Proof. Let $f: V(T)-\{\operatorname{root}(T)\} \rightarrow V(G)$ be a mapping satisfying

(i) if $t^{\prime}$ is a successor of $t$ then $f\left(t^{\prime}\right) \in W_{t^{\prime}}-W_{t}$, and

(ii) if $t \neq \operatorname{root}(T)$ does not have a predecessor then $f(t) \in W_{t^{\prime}}-W_{t}$, where $t^{\prime}$ is a successor of $t$.

Such a mapping exists by (S2) and (S3). It follows from (W2) that $\left|f^{-1}(v)\right| \leq 2$ for every $v \in V(G)$, and hence $|V(T)| \leq|V(G)|$ because $G$ is infinite.

To prove the assertion about width we must prove that

(1) for every chain $C \subseteq V(T)$, the set $W_{C}=\bigcup_{t \in C} \bigcap\left\{W_{t^{\prime}}: t^{\prime} \in C, t^{\prime} \geq t\right\}$ has cardinality $<\kappa$.

We prove (1) by transfinite induction on the ordinal type of the set $\left\{t^{\prime} \in\right.$ $V(T): t^{\prime} \leq t$ for some $\left.t \in C\right\}$, which we denote by $h(C)$. From (S1) we deduce that (1) is true if $h(C) \leq 1$. So assume that (1) is true for all $C$ with $h(C)<\alpha$ and let $C$ with $h(C)=\alpha>1$ be given. If $C$ has a maximal element, say $t$, and $t$ does not have a predecessor, then $W_{C}=W_{t}=W_{C-\{t\}}$ by (S3), which has cardinality $<\kappa$ by the induction hypothesis. If $t$ has a predecessor, say $t^{\prime}$, then $W_{C}=W_{t}, W_{C-\{t\}}=W_{t^{\prime}}$ and $\left|W_{t}\right|<\kappa$ by $(\mathrm{S} 2)$ and the induction hypothesis. Finally, if $C$ does not have a maximal element, then $\left|W_{C}\right|<\kappa$ by (S3) and (2.3).

This proves (1) and hence (2.4).

If $T=(V, \leq)$ is a well-founded tree and $t \in V$, then the order type of the set $\left\{t^{\prime} \in V: t^{\prime}<t\right\}$ is called the height of $t$ and is denoted by $h t(t)$. The following implies that if $\kappa=\aleph_{0}$ then the well-founded tree-decomposition in (ii) of (1.4) can be chosen graph-theoretic.

(2.5) Let $\kappa$ be an infinite regular cardinal, and let $G$ and $(T, W)$ be as in (2.4). Then every chain in $T$ has order type $\leq \kappa$.

Proof. Suppose not. Then there is a vertex $t_{0} \in V(T)$ with $\operatorname{ht}\left(t_{0}\right)=\kappa$. By (S3) there exists a successor $t_{0}^{\prime}$ of $t_{0}$, and by $(\mathrm{S} 2)$ there exists a $W_{t_{0}}$-flap $F$ with $F \cap W_{t_{0}^{\prime}} \neq \varnothing$. For every $v \in W_{t_{0}}$ there exists, by (S3), $t_{v}<t_{0}$ such that $v \in W_{t}$ for all $t$ with $t_{v} \leq t \leq t_{0}$. Let $t_{1}=\sup \left\{t_{v}: v \in W_{t_{0}}\right\}$. Since $\left|W_{t_{0}}\right|<\kappa$ by (2.4) and since $\kappa$ is regular, we deduce that $t_{1}$ is well-defined and that $t_{1}<t_{0}$. Since $W_{t_{0}} \subseteq W_{t_{1}}$, it follows that either $F \cap W_{t_{1}} \neq \varnothing$, or $F$ is a $W_{t_{1}}$-flap in which case $F \cap W_{t_{1}^{\prime}} \neq \varnothing$ for some successor $t_{1}^{\prime}$ of $t_{1}$, by (S4). In either case, $F \cap W_{t_{0}} \neq \varnothing$ by (1.7), a contradiction.

Our next result implies (i) $\Rightarrow$ (iii) of (1.4) for regular uncountable cardinals and thus completes the proof of (1.4).

(2.6) Let $\kappa$ be a regular uncountable cardinal, and let $G$ and $(T, W)$ be as in (2.4). Then $T$ is $<\kappa$ high.

Proof. Suppose for a contradiction that $C$ is a chain in $T$ of order type $\kappa$. We may assume that $C$ has the property that if $t \in C$ and $t^{\prime} \leq t$ then $t^{\prime} \in C$. 
Let $X=\bigcup_{t \in C} \bigcap\left\{W_{t^{\prime}}: t^{\prime} \in C, t^{\prime} \geq t\right\}$. From (2.4), $|X|<\kappa$, and since $\kappa$ is regular it follows that there exists $t_{0} \in C$ such that $X \subseteq W_{t}$ for every $t \in C$ with $t_{0} \leq t$.

(1) For every $t_{1} \in C$ with $t_{1} \geq t_{0}$ there exists $t \in C$ with $t \geq t_{1}$ such that $X=W_{t}$.

To prove (1) we shall construct a sequence $t_{1}<t_{2}<\cdots$ of elements of $C$ as follows. Assume that $n \geq 1$ and that $t_{n}$ has already been constructed. We may assume that $W_{t_{n}}-X \neq \varnothing$, for otherwise we are done. For every $v \in W_{t_{n}}-X$ there exists $t_{v} \in C$ with $t_{v} \geq t_{n}$ and such that $v \notin W_{t_{v}}$. Since $\left|W_{t_{n}}\right|<\kappa$ and $\kappa$ is regular, it follows that $t_{n+1}:=\sup \left\{t_{v}: v \in W_{t_{n}}-X\right\}$ exists and belongs to $C$, and that $\left(W_{t_{n}}-X\right) \cap W_{t_{n+1}}=\varnothing$. Since $W_{t_{n}}-X \neq \varnothing$ we deduce that $t_{n+1}>t_{n}$. Since $\kappa$ is regular uncountable it follows that $t=\sup t_{n}$ exists and belongs to $C$. We claim that $t$ is as desired. For suppose for a contradiction that $v \in W_{t}-X$. Then $v \in W_{t^{\prime}}$ for some $t^{\prime}<t$ by (S3), and hence $v \in W_{t_{n}}$ for some $n$. But then $v \notin W_{t_{n+1}}$, contrary to (W2). Hence such a choice of $v$ is impossible, which proves (1).

From (1) we deduce that there exist $t_{1}, t_{2} \in C$ with $t_{0} \leq t_{1}<t_{2}$ such that $W_{t_{1}}=W_{t_{2}}=X$. Let $t_{2}^{\prime} \in C$ be the successor of $t_{2}$ in $C$. By (S2), $W_{t_{2}^{\prime}} \cap F \neq \varnothing$ for some $X$-flap $F$ of $G$. By (S4), $W_{t_{1}^{\prime}} \cap F \neq \varnothing$ for some successor $t_{1}^{\prime}$ of $t_{1}$. But $X \cap F=W_{t_{2}} \cap F \neq \varnothing$ by (1.7), a contradiction.

A natural question arises whether a highly connected graph can be decomposed into highly connected pieces. This turns out to be true, as follows. If $\mu$ is a cardinal we say that a graph $G$ is $\mu$-connected if $|V(G)| \geq \mu$ and $G \backslash X$ is connected for every $X \subseteq V(G)$ with $|X|<\mu$. We say that a subset $A \subseteq V(G)$ is $\mu$-connected if the graph induced by $A$ is $\mu$-connected.

(2.7) Let $\kappa$ be a regular uncountable cardinal, let $\mu<\kappa$ be an infinite cardinal, and let $G$ be a $\mu$-connected graph which contains no subgraph isomorphic to a subdivision of $K_{\kappa}$. Then there exists a well-founded tree-decomposition $(T, W)$ of $G$ such that

(i) $|V(T)| \leq|V(G)|$,

(ii) every chain of $T$ has order type $<\kappa$,

(iii) $\left|W_{t}\right|<\kappa$ and $W_{t}$ is $\mu$-connected for every $t \in V(T)$, and

(iv) for $t, t^{\prime} \in V(T)$, if $t^{\prime} \leq t$ then $W_{t^{\prime}} \subseteq W_{t}$, and if $\operatorname{ht}(t)$ is a limit ordinal then $W_{t}=\bigcup_{t^{\prime}<t} W_{t^{\prime}}$.

Proof. Let $\left(T, W^{\prime \prime}\right)$ be a standard decomposition of $G$. Then by $(2.4),|V(T)|$ $\leq|V(G)|$ and $\left(T, W^{\prime \prime}\right)$ has width $<\kappa$, and by (2.6) every chain in $T$ has order type $<\kappa$. Since $G$ is $\mu$-connected there exists a $\mu$-connected set $M \subseteq V(G)$ with $|M|=\mu$. For $t \in V(T)$ let $W_{t}=W_{t}^{\prime \prime} \cup M$, and let $W=\left(W_{t}: t \in \bar{V}(T)\right)$. Then $(T, W)$ is a well-founded tree-decomposition of $G$ of width $<\kappa$.

(1) Let $t_{1} \in V(T)$ be a successor of $t_{0} \in V(T)$. There is a subset $D_{t_{1}} \subseteq$ $\bigcup_{t \geq t_{1}} W_{t}$ such that

(i) $W_{t_{1}} \subseteq D_{t_{1}}$ and $\left|D_{t_{1}}\right|<\kappa$, and

(ii) there is no cut $(A, B)$ of the restriction of $G$ to $D_{t_{1}}$ such that $|A \cap B|<$ $\mu, A \nsubseteq B \nsubseteq A$, and $W_{t_{0}} \cap W_{t_{1}} \subseteq A$.

For since $G$ is $\mu$-connected there is a $\mu$-connected $F \subseteq V(G)$ with $W_{t_{1}} \subseteq F$ and with $|F|=\max \left(\mu,\left|W_{t_{1}}\right|\right)$. Set $D_{t_{1}}=F \cap \bigcup_{t \geq t_{1}} W_{t}$; then (i) is clearly 
satisfied. To prove that $D_{t_{1}}$ satisfies (ii) suppose for a contradiction that $(A, B)$ is a cut as in (ii). Since the restriction of $G$ to $F$ is $\mu$-connected there exists a path $P$ in $G$ joining a vertex of $A-B$ to a vertex of $B-A$ with $V(P) \subseteq$ $F-(A \cap B)$. Let us choose such a path with $|V(P)|$ minimum. Since $(A, B)$ is a cut of the restriction of $G$ to $D_{t_{1}}$ it follows that $V(P)-\bigcup_{t \geq t_{1}} W_{t} \neq \varnothing$. From (W1) and (1.7) it follows that $P$ contains a proper subpath joining a vertex $u \in W_{t_{0}} \cap W_{t_{1}} \subseteq A$ to a vertex in $B-A$. But $u \notin B$ and this subpath is shorter than $P$, a contradiction. Thus $D_{t_{1}}$ satisfies both (i) and (ii) and the proof of (1) is complete.

Let $D_{\text {root }(T)}=M$, and for each $t \in V(T)-\{\operatorname{root}(T)\}$ with no predecessor, let $D_{t}=\varnothing$. For each $t \in V(T)$ let $W_{t}^{\prime}=\bigcup_{t^{\prime} \leq t} D_{t^{\prime}}$.

(2) For each $t \in V(T),\left|W_{t}^{\prime}\right|<\kappa$ and $W_{t}^{\prime}$ is $\mu$-connected.

For since every chain of $T$ has order type $<\kappa$ and each $\left|D_{t^{\prime}}\right|<\kappa$ and $\kappa$ is regular, it follows that each $\left|W_{t}^{\prime}\right|<\kappa$. We prove that $W_{t}^{\prime}$ is $\mu$-connected by transfinite induction on $\mathrm{ht}(t)$. Certainly $\left|W_{t}^{\prime}\right| \geq \mu$ since $D_{\text {root }(T)} \subseteq W_{t}^{\prime}$. If $t$ is a successor of some $t_{0}$ then $W_{t}^{\prime}=W_{t_{0}}^{\prime} \cup D_{t}$. Suppose that $(A, B)$ is a cut of the restriction of $G$ to $W_{t}^{\prime}$ with $|A \cap B|<\mu$ and $A-B, B-A \neq \varnothing$. Now from the inductive hypothesis, $W_{t_{0}}^{\prime}$ is $\mu$-connected, and so not both $(A-B) \cap W_{t_{0}}^{\prime}$, $(B-A) \cap W_{t_{0}}^{\prime} \neq \varnothing$. We assume that $W_{t_{0}}^{\prime} \subseteq A$. Now $\left(A \cap D_{t}, B \cap D_{t}\right)$ is a cut of the restriction of $G$ to $D_{t}$, and certainly $B \cap D_{t} \subseteq A \cap D_{t}$ since $B \nsubseteq A$ and $W_{t_{0}}^{\prime} \subseteq A$. From the choice of $D_{t}$ it follows that $A \cap D_{t} \subseteq B \cap D_{t}$, and so $D_{t} \subseteq B$. Thus $W_{t_{0}} \cap W_{t} \subseteq W_{t_{0}}^{\prime} \cap D_{t} \subseteq A \cap B$, and so $\left|W_{t_{0}} \cap W_{t}\right|<\mu$. But $M \subseteq W_{t_{0}} \cap W_{t}$, a contradiction. Finally, suppose that $t$ has no predecessor. Since $W_{\operatorname{rot}(T)}^{\prime}$ is $\mu$-connected we may assume that $t \neq \operatorname{root}(T)$. Let $C=\left\{t^{\prime} \in V(T): t^{\prime}<t\right\}$. Then $W_{t}^{\prime}=\bigcup_{t^{\prime} \in C} W_{t^{\prime}}^{\prime}$ since $D_{t}=\varnothing$, and so $W_{t}^{\prime}$ is $\mu$-connected since it is the union of a nested sequence of $\mu$-connected subsets. This proves (2).

Let $W^{\prime}=\left(W_{t}^{\prime}: t \in V(T)\right)$.

(3) $\left(T, W^{\prime}\right)$ is a well-founded tree-decomposition.

We observe first that $W_{t} \subseteq W_{t}^{\prime}$ for each $t \in V(T)$. This is clear if $t=$ $\operatorname{root}(T)$ or $t$ is a successor. Otherwise let $C$ be the set of all $t^{\prime}<t$ which are successors. Then $W_{t} \subseteq \bigcup_{t^{\prime} \in C} W_{t^{\prime}}$ by (S3); each such $W_{t^{\prime}} \subseteq W_{t^{\prime}}^{\prime}$, and $\bigcup_{t^{\prime} \in C} W_{t^{\prime}}^{\prime} \subseteq W_{t}^{\prime}$ by the definition of $W^{\prime}$. This proves that $W_{t} \subseteq W_{t}^{\prime}$ for each $t \in V(T)$ and (W1) follows. To see (W2), let $t_{1}, t_{2}, t_{3} \in V(T)$ with $t_{2} \in T\left[t_{1}, t_{3}\right]$; and let $v \in W_{t_{1}}^{\prime} \cap W_{t_{3}}^{\prime}$. Choose $t_{1}^{\prime} \leq t_{1}$ with $v \in D_{t_{1}^{\prime}}$, and $t_{3}^{\prime} \leq t_{3}$ similarly. If $t_{1}^{\prime} \leq t_{2}$ or $t_{3}^{\prime} \leq t_{2}$ then $v \in W_{t_{2}}^{\prime}$ as required, and so we assume not. Hence $t_{2} \in T\left[t_{1}^{\prime}, t_{3}^{\prime}\right]$. Now $v \in D_{t_{1}^{\prime}} \subseteq \bigcup_{t \geq t_{1}^{\prime}} W_{t}$, and similarly $v \in \bigcup_{t \geq t_{3}^{\prime}} W_{t}$. Since $t_{2} \in T\left[t_{1}^{\prime}, t_{3}^{\prime}\right]$ and $t_{2} \leq t_{1}^{\prime}, t_{3}^{\prime}$ it follows from (W2) (for $(T, W)$ ) that $v \in W_{t_{2}} \subseteq W_{t_{2}}^{\prime}$ as required. Condition (W3) is clear because $W_{t}^{\prime} \subseteq W_{t^{\prime}}^{\prime}$ for $t \leq t^{\prime}$.

Finally, we observe that if $t \in V(T)-\{\operatorname{root}(T)\}$ has no predecessor (i.e., $\mathrm{ht}(t)$ is a limit ordinal) then since $D_{t}=\varnothing$,

$$
W_{t}^{\prime}=\bigcup_{t^{\prime}<t} D_{t^{\prime}}=\bigcup_{t^{\prime}<t} W_{t^{\prime}}
$$

The result follows.

For an application of (2.7) see [9]. 


\section{LINEAR DECOMPOSITIONS AND STOPPAGES}

Now we prove (1.5). By (1.4), (i) $\Rightarrow$ (ii). We shall prove (ii) $\Rightarrow$ (iii) $\Rightarrow$ (iv) $\Rightarrow$ (i). We start with (ii) $\Rightarrow$ (iii) .

(3.1) Let $\kappa$ be an uncountable regular cardinal, let $G$ be a graph and let $(T, W)$ be $a<\kappa$ high well-founded tree-decomposition of $G$ of width $<\kappa$. Then $G$ admits a linear decomposition of width $<\kappa$.

Proof. Let $\leq^{\prime}$ be a well-ordering of $V(T)$ with the property that

(1) for every $t \in V(T)$, the order type of the set of successors of $t$ is not a limit ordinal.

Let $L$ be the set of maximal chains of $T$ and let $\leq$ be defined on $L$ as follows. For $l_{1}, l_{2} \in L$ we say that $l_{1} \leq l_{2}$ if either $l_{1}=l_{2}$, or $l_{1} \neq l_{2}$ and $t_{1} \leq^{\prime} t_{2}$, where $t_{i}=\min \left\{t \in l_{i}: t \notin l_{1} \cap l_{2}\right\}$. It follows that $L$ is linearly ordered by $\leq$.

(2) $(L, \leq)$ is complete.

We must verify that every nonempty subset of $L$ has a supremum and an infimum. We shall do it for supremum, for the infimum case is analogous and in fact easier. So let $C \subseteq L$. Let $t_{0}=\operatorname{root}(T)$ and assume that we have already constructed $t_{0}, \ldots, t_{\beta}, \ldots(\beta<\alpha)$, where $\mathrm{ht}\left(t_{\beta}\right)=\beta$ and $t_{0} \leq t_{1} \leq \cdots \leq t_{\beta} \leq$ $\cdots$. If $\left\{t_{\beta}\right\}_{\beta<\alpha}$ is a maximal chain in $T$ we stop, and otherwise we choose $t_{\alpha} \in V(T)$ such that

(i) $\operatorname{ht}\left(t_{\alpha}\right)=\alpha$,

(ii) $t_{\beta} \leq t_{\alpha}$ for every $\beta<\alpha$,

(iii) $t \leq t_{\alpha}$ for every $t$ such that $h t(t)=\alpha, t_{\beta} \leq t$ for every $\beta<\alpha$, and $t \in l$ for some $l \in C$,

(iv) $t_{\alpha}$ is $\leq^{\prime}$-minimal subject to (i)-(iii).

Such a choice is possible, because it follows from (1) that there exists at least one vertex satisfying (i)-(iii). (We remark that this vertex is unique when $\alpha$ is a limit ordinal, because any two vertices of $T$ have an infimum.) Let $\alpha$ be the least ordinal such that $t_{\alpha}$ is undefined, and let $l=\left\{t_{\beta}: \beta<\alpha\right\}$. Then $l=\sup C$, as is easily seen. This proves $(2)$.

We define, for $l \in L, X_{l}=\bigcup_{t \in l} W_{t}$, and put $X=\left(X_{l}: l \in L\right)$. We claim that $(L, X)$ is a linear decomposition of $G$ of width $<\kappa$. For condition (L1) is obviously satisfied. To prove (L2) let $l_{1}<l<l_{2}$ and let $v \in X_{l_{1}} \cap X_{l_{2}}$. Then $v \in W_{t_{1}} \cap W_{t_{2}}$ for some $t_{1} \in l_{1}$ and some $t_{2} \in l_{2}$. Let $t=\inf \left(t_{1}, t_{2}\right)$; then $t \in l$ and so $W_{t} \subseteq X_{l}$, but $v \in W_{t}$ by (W2) and so $v \in X_{l}$, as required. To prove (L3) let $I \subseteq L$ be a nonempty interval. Again, it is enough to show that $\bigcap_{i \in I} X_{i} \subseteq$ $X_{\sup (I)}$. So let $l=\sup I$ and let $i_{1} \in I$; we may assume that $i_{1} \neq l$. Then there exists $i_{2} \in I$ such that $i_{1} \cap i_{2} \subseteq l$. Let $t_{j}=\inf \left\{t \in V(T): t \in i_{j}-i_{3-j}\right\}$ $(j=1,2)$ and let $t=\inf \left(t_{1}, t_{2}\right)$. Then $\bigcap_{i \in I} X_{i} \subseteq X_{i_{1}} \cap X_{i_{2}} \subseteq \bigcup_{t^{\prime} \leq t} W_{t^{\prime}} \subseteq X_{l}$ using (W2), as desired.

Hence $(L, X)$ is a linear decomposition. The statement about width follows easily, because $\kappa$ is regular and each $X_{l}$ is the union of $<\kappa$ sets, each of cardinality $<\kappa$.

(3.2) Let $\kappa$ be a cardinal and let $G$ be a graph. If $G$ admits a linear decomposition of width $<\kappa$, then $G$ has no stoppage of order $\geq \kappa$.

Proof. Suppose for a contradiction that $(L, X)$ is a linear decomposition of 
$G$ of width $<\kappa$ and that $\mathscr{S}$ is a stoppage in $G$ of order $\geq \kappa$. For $l \in L$ let $C_{l}=\left(\bigcup_{l^{\prime} \leq l} X_{l^{\prime}}, \bigcup_{l^{\prime} \geq l} X_{l^{\prime}}\right)$. We observe that $C_{l}$ is a cut in $G$ of order $<\kappa$. Let $A$ be the set of all $l \in L$ such that $C_{l} \in \mathscr{S}$. We deduce from the second stoppage axiom that if $l \in A$ and $l^{\prime} \leq l$, then $l^{\prime} \in A$.

(1) $A$ has a maximum element.

For let $a=\sup A$. We deduce from the third stoppage axiom that

$$
\left(\bigcup_{l<a} X_{l}, \bigcup_{l \geq a} X_{l}\right) \in \mathscr{S} \text {. }
$$

If $\left(\bigcup_{l \leq a} X_{l}, \bigcup_{l \geq a} X_{l}\right) \notin \mathscr{S}$ then $\left(\bigcup_{l \geq a} X_{l}, \bigcup_{l \leq a} X_{l}\right) \in \mathscr{S}$ by the first stoppage axiom, and yet $\left(G \mid \cup_{l<a} X_{l}\right) \cup\left(G \mid \cup_{l \geq a} X_{l}\right)=G$, contrary to the second axiom. Hence $\left(\bigcup_{l \leq a} X_{l}, \bigcup_{l \geq a} X_{l}\right) \in \mathscr{S}$ and thus $a \in A$.

Similarly let $B$ be the set of all $l \in L$ such that $\left(\bigcup_{l^{\prime} \geq l} X_{l^{\prime}}, \bigcup_{l^{\prime} \leq l} X_{l^{\prime}}\right) \in \mathscr{S}$. Analogously, $B$ has a minimum element, say $b$. It follows that $a<b$ and that there is no $l \in L$ with $a<l<b$. Hence $\left(G \mid \bigcup_{l \leq a} X_{l}\right) \cup\left(G \mid \bigcup_{l \geq b} X_{l}\right)=G$, contrary to the second stoppage axiom.

(3.3) Let $\kappa$ be an infinite cardinal, let $G$ be a graph and let $H$ be a subgraph of $G$ isomorphic to a subdivision of $K_{\kappa}$. Then $G$ has a stoppage of order $\kappa$.

Proof. Let $V$ be the set of vertices of $H$ which correspond to a vertex of $K_{\kappa}$. If $(A, B)$ is a cut in $G$ of order $<\kappa$, then exactly one of $A, B$ contains $V$. Let $\mathscr{S}$ be the set of all cuts $(A, B)$ in $G$ of order $<\kappa$ such that $V \subseteq B$. Then it is easy to verify that the stoppage axioms are satisfied.

Thus we have proved (1.5). Now we prove (1.6) which we restate.

(3.4) There exists a graph which contains no subgraph isomorphic to a subdivision of $K_{\aleph_{\omega}}$ and which has no linear decomposition of width $<\aleph_{\omega}$.

Proof. Let $T$ be the well-founded tree with every chain of order type $\leq \omega$ in which every element $t \in V(T)$ has $\aleph_{\omega}$ successors. For every $t \in V(T)$ we choose a set $M_{t}$ and an element $m_{t} \in M_{t}$ in such a way that $\left|M_{t}\right|=\aleph_{\mathrm{ht}(t)}$ and $M_{t} \cap M_{t^{\prime}}=\varnothing$ for distinct $t, t^{\prime} \in V(T)$. Let $G$ be the simple graph with vertex set $\bigcup_{t \in V(T)} M_{t}$ and such that for $u, v \in V(G)$ there exists an edge in $G$ with endvertices $u, v$ if and only if $u \neq v$, and either $u, v \in M_{t}$ for some $t \in V(T)$, or $u \in M_{t}$ and $v=m_{t^{\prime}}$ for a successor $t^{\prime} \in V(T)$ of $t \in V(T)$, or $v \in M_{t}$ and $u=m_{t^{\prime}}$ for a successor $t^{\prime} \in V(T)$ of $t \in V(T)$. For $t \in V(T)$ let $G_{t}$ be the subgraph of $G$ induced by the set $\bigcup_{t^{\prime}>t} M_{t^{\prime}}$. Let $W_{\operatorname{root}(T)}=M_{\text {root }(T)}$, and if $t^{\prime} \in V(T)$ is a successor of $t \in V(T)$ let $W_{t^{\prime}}=M_{t} \cup M_{t^{\prime}}$. Let $W=\left(W_{t}: t \in V(T)\right)$. It is easy to see that $(T, W)$ is a well-founded tree-decomposition of $G$ of width $<\aleph_{\omega}$, and hence $G$ has no subgraph isomorphic to a subdivision of $K_{\aleph_{\omega}}$ by (1.4).

It remains to be shown that $G$ has no linear decomposition of width < $\aleph_{\omega}$ either. So suppose for a contradiction that $G$ has a linear decomposition $(L, X)$ of width $\left\langle\aleph_{\omega}\right.$. A clique in $G$ is a subset $M \subseteq V(G)$ such that every pair of distinct members of $M$ are adjacent.

(1) For every clique $M$, the set $I(M)=\left\{l \in L: M \subseteq X_{l}\right\}$ is a nonempty closed interval in $L$. 
For $v \in M, I(\{v\})$ is a closed interval by (L2) and (L3), and by (L1) any two of these intervals meet. We deduce that $\left\{l \in L: M \subseteq X_{l}\right\}=\bigcap_{v \in M} I(\{v\})$ is as desired.

For $t \in V(T)$ let $a(t) \leq b(t)$ be the endvertices of $I\left(M_{t}\right)$. Let $t_{0}=\operatorname{root}(T)$ and assume that we have already constructed $t_{0}, \ldots, t_{n}$. Let $t_{n+1}$ be a successor of $t_{n}$ such that $M_{t_{n+1}} \cap\left(X_{a\left(t_{n}\right)} \cup X_{b\left(t_{n}\right)}\right)=\varnothing$. Such a choice is possible since $\left|X_{a\left(t_{n}\right)} \cup X_{b\left(t_{n}\right)}\right|<\aleph_{\omega}$ and there are $\aleph_{\omega}$ such successors. This completes the inductive definition of $t_{0}, t_{1}, \ldots$.

Now for each $n \geq 0, M=M_{t_{n}} \cup\left\{m_{t_{n+1}}\right\}$ is a clique, and so $\varnothing \neq I(M)=$ $I\left(M_{t_{n}}\right) \cap I\left(\left\{m_{t_{n+1}}\right\}\right)$. Yet $a\left(t_{n}\right), b\left(t_{n}\right) \notin I\left(\left\{m_{t_{n+1}}\right\}\right)$, and hence $I\left(\left\{m_{t_{n+1}}\right\}\right) \subseteq$ $I\left(M_{t_{n}}\right)$. But $I\left(M_{t_{n+1}}\right) \subseteq I\left(\left\{m_{t_{n+1}}\right\}\right)$, and so $I\left(M_{t_{n+1}}\right) \subseteq I\left(M_{t_{n}}\right)$. By the completeness of $L$ there exists $l \in L$ such that $l \in \bigcap_{n \geq 0} I\left(M_{t_{n}}\right)$, that is, $M_{t_{n}} \subseteq X_{l}$ for all $n \geq 0$. But $\left|M_{t_{0}} \cup M_{t_{1}} \cup \cdots\right|=\aleph_{\omega}>\left|X_{l}\right|$, a contradiction.

\section{REFERENCES}

1. D. Bienstock, N. Robertson, P. D. Seymour, and R. Thomas, Quickly excluding a forest, J. Combin. Theory Ser. B 52 (1991), 274-283.

2. R. Diestel, Graph decompositions, Oxford University Press, Oxford 1990.

3. $\mathrm{R}$ Halin, Unterteilungen vollständiger Graphen in Graphen mit unendlicher chromatische Zahl, Abh. Math. Sem. Univ. Hamburg 31 (1967), 156-165.

4. I. Křiž and R. Thomas, Clique-sums, tree-decompositions and compactness, Discrete Math. 81 (1990), 177-185.

5. N. Robertson and P. D. Seymour, Graph minors. I. Excluding a forest, J. Combin. Theory Ser. B 35 (1983), 39-61.

6. __ Graph minors. III. Planar tree-width, J. Combin. Theory Ser. B 36 (1984), 49-63.

7. N. Robertson, P. D. Seymour, and R. Thomas, Excluding infinite minors, Discrete Math. 95 (1991), 303-319.

8. P. D. Seymour and R. Thomas, Excluding infinite trees, Trans. Amer. Math. Soc. (to appear).

9. __ An end-faithful spanning tree counterexample, Proc. Amer. Math. Soc. 113 (1991), 1163-1171.

Department of Mathematics, Ohio State University, Columbus, Ohio 43210

E-mail address: robertso@function.mps.ohio-state.edu

Bellcore, 445 South Street, Morristown, New Jersey 07962

E-mail address: pds@breeze.bellcore.com

School of Mathematics, Georgia Institute of Technology, Atlanta, Georgia 30332

E-mail address: thomas@math.gatech.edu 